Abstract S14 Table 1 Demopgraphic and clinical data of study patients. Data are summarised as number (proportion) mean \pm standard deviation, or median [inter quartile range]

\begin{tabular}{|l|c|c|}
\hline Variable & Group A & Group B \\
\hline Age, years & $41.3 \pm 13.8$ & $49.6 \pm 14.2$ \\
\hline Sex, female & $7 / 18(38.9 \%)$ & $6 / 18(33.3 \%)$ \\
\hline RAPID score & $2[1-3.25]$ & $3[1.75-4]$ \\
\hline Length of hospital stay, days & $10.25 \pm 3.32$ & $11.44 \pm 2.64$ \\
\hline Time to from discharge to return & $10[4.5-18]$ & $14.5[4.5-18.5]$ \\
to normal level of activity, days & $(\mathrm{N}=8)$ & $(\mathrm{n}=8)$ \\
\hline Infection relapse, $\mathrm{n}$ & $3 / 17((17.6 \%)$ & $2 / 17(11.7 \%)$ \\
\hline
\end{tabular}

diagnosis, a three-week antibiotic course appears to be sufficient.

Registration NCT04615286

\section{S15 EVOLUTION OF MESOTHELIOMA FOLLOWING INITIAL BIOPSIES SHOWING BENIGN PLEURAL INFLAMMATION: A META-ANALYSIS}

${ }^{1} \mathrm{KJ}$ Ferguson, ${ }^{2} \mathrm{KG}$ Blyth, ${ }^{3} \mathrm{M}$ Neilson. ${ }^{1}$ Pleural Disease Unit, Glasgow, UK; ${ }^{2}$ Institute of Cancer Sciences, University of Glasgow, UK; ${ }^{3}$ Cancer Research UK Beatson Institute, Glasgow, UK

\subsection{6/thorax-2021-BTSabstracts.21}

Introduction Malignant Pleural Mesothelioma (MPM) is typically preceded by chronic pleural inflammation, providing a unique window for translational research. Within the PREDICT-Meso International Accelerator Network, the Meso-ORIGINS (Mesothelioma Observational study of RIsk prediction and Generation of benign-meso tissue pairs, Including a Nested MRI Sub-study) study will recruit 500 asbestosexposed patients with initial benign pleural biopsies. All will undergo detailed surveillance at $\sim 20$ UK sites, including repeat biopsies in the minority who evolve into MPM. These paired tissue samples will be used to define new therapeutic targets and develop new pre-clinical models for drug screening. Here, we integrate data from the 4-centre Meso-ORIGINS feasibility study regarding Benign-MPM evolution rate, with previously published literature. This data is being used to finalise the Meso-ORIGINS study design and site selection.

Methods Studies were identified on PubMed using the search terms 'non-specific pleuritis', 'benign fibrinous pleurisy' and 'mesothelioma'. The following data were extracted: publication year, number of benign pleuritis cases, number of subsequent evolutions, cohort entry criteria (including biopsy technique, asbestos exposure), median follow-up, country and region of origin, and study design (retrospective or prospective). A

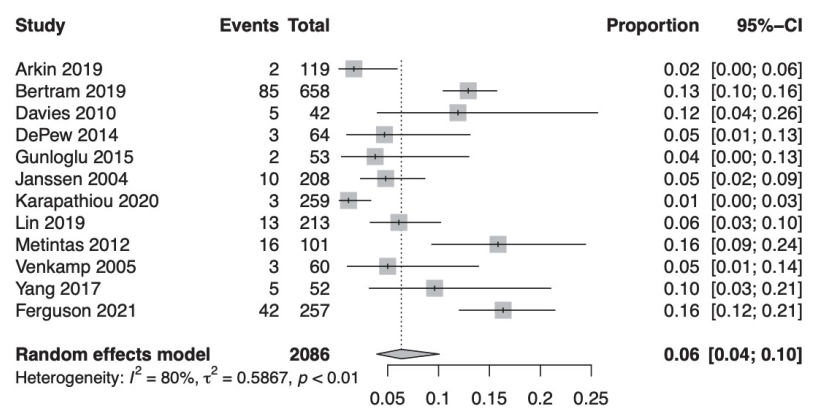

Abstract S15 Figure 1 random effects meta-analysis model was used to analyse the primary outcome; MPM evolution rate, with $\mathrm{I}^{2}$ used to assess between-study heterogeneity.

Results 11 studies were identified. These data were combined with Meso-ORIGINS feasibility study data (42 evolutions from 257 benign cases (16\%)), ${ }^{1}$ generating a total of 189 evolutions from 2086 benign cases. The summary point estimate of MPM evolution was $6 \%$ (95\% CI 4-10), see figure 1 , which also confirms significant between study heterogeneity $\left(\mathrm{I}^{2} 80 \%, \mathrm{p}<0.01\right)$.

Conclusion The Benign-MPM evolution rate varies in the reported studies, which show high inter-study heterogeneity. Sub-group analyses of individual study factors associated with higher MPM evolution rates are ongoing, including biopsy techniques (LAT vs VATS), median follow-up, geographical area in relation to MPM incidence and asbestos exposure frequency. These data will inform the site selection process during Meso-ORIGINS set-up.

\section{REFERENCE}

1. Ferguson K, Mercer R, King J, et al. S43 Preliminary results of the Meso-ORIGINS feasibility study: retrospective element regarding BAPE-mesothelioma evolution rate. Thorax 2021;76:A28.

\section{Predictive tools for acute deterioration in COVID-19 and beyond}

\section{S16 PROGNOSTICATION IN HOSPITAL ACQUIRED PNEUMONIA - ARE CURRENT SCORING SYSTEMS FIT FOR PURPOSE?}

\begin{abstract}
${ }^{1,2} \mathrm{~A}$ Adiga, ${ }^{2,3} \mathrm{~F}$ Grudzinska, ${ }^{2,3,4} \mathrm{D}$ Dosanjh, 2,3,4,5 D Parekh. 'Birmingham Medical School, University of Birmingham, Birmingham, UK; ${ }^{2}$ Birmingham Acute Care Research Group, Institute of Inflammation and Ageing, University of Birmingham, Birmingham, UK; ${ }^{3}$ Department of Respiratory Medicine, Queen Elizabeth Hospital Birmingham, University Hospitals Birmingham NHS Foundation Trust, Birmingham, UK; ${ }^{4}$ Birmingham Respiratory Clinical Trials Unit, Birmingham, UK; ${ }^{5}$ Department of Critical Care Medicine, Queen Elizabeth Hospital Birmingham, University Hospitals Birmingham NHS Trust, Birmingham, UK
\end{abstract}

\subsection{6/thorax-2021-BTSabstracts.22}

Introduction Non-ventilator hospital acquired pneumonia (NVHAP) is the most common nosocomial infection in England. Evidence regarding prognostic factors in NV-HAP patients is limited. No NV-HAP-specific scoring systems that can identify patients at risk of poor outcomes are in widespread use. This study aims to identify factors associated with mortality and assess the accuracy of currently used scoring systems in NVHAP patients.

Methods Diagnostic criteria for NV-HAP were adapted from the definition described in the 2016 American Thoracic Society/Infectious Diseases Society of America guidelines. Case notes of adult patients admitted to a tertiary hospital between 22nd November 2019-23rd February 2020 with an International Classification of Diseases (ICD)-10 coded diagnosis of NV-HAP were retrospectively screened. For patients that met the diagnostic criteria, clinical variables at the time of HAP diagnosis were recorded and four prognostic scores were calculated. The scoring systems assessed were: CURB65; National Early Warning Score 2 (NEWS2); Quick Sequential Organ Failure Assessment (qSOFA); and the Japanese Respiratory Society Immunodeficiency, Respiration, Orientation, Age, Dehydration (IROAD) score. The association between clinical variables and 30-day mortality was investigated using 


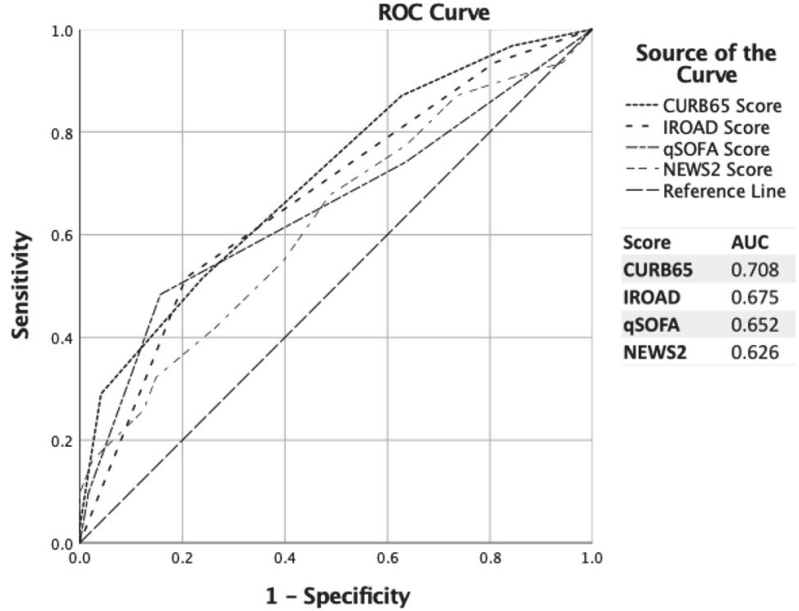

Abstract S16 Figure 1 Receiver operating characteristic (ROC) curves and area under the curve (AUC) values for 30-day mortality prediction by CURB65, IROAD, qSOFA and NEWS2 scoring systems

univariate analysis. The accuracy of the scoring systems in predicting 30-day mortality was assessed using receiver operating characteristic (ROC) analysis.

Results We identified 323 patients with an ICD-10 coded diagnosis of NV-HAP. 154/323 (48\%) met the diagnostic criteria for inclusion. 30-day mortality in these included patients was $21.4 \%$. Univariate analysis revealed advanced age, emergency admission, speech/swallowing difficulties, altered mental status, lymphocyte count $<0.7 \times 10^{9} / \mathrm{L}$, neutrophil-to-lymphocyte-ratio $\geq 10$, urea $>7 \mathrm{mmol} / \mathrm{L}$, acute kidney injury, CURB65 $\geq 2, \mathrm{qSOFA} \geq 2$ and $\mathrm{IROAD}=$ 'severe' as being significantly associated with 30-day mortality. Raised white blood cell count, C-reactive protein, the extent of radiographic features, prior antibiotic therapy and NEWS $2>4$ were not significantly associated with mortality. In ROC analysis, scoring systems achieved the following area under the curve values: CURB65-0.708; IROAD-0.675; qSOFA-0.652 and NEWS2-0.626 (figure 1).

Conclusion NV-HAP-specific scoring systems are needed, as current scoring systems have limited prognostic accuracy. Larger studies with the power to perform multivariate analysis are required to validate the factors identified as being associated with mortality. These factors may be used to create a more accurate, NV-HAP-specific scoring system.

\section{S17 DYNAMIC EARLY WARNING SCORE VERSUS NATIONAL EARLY WARNING SCORE-2 FOR PREDICTING CLINICAL DETERIORATION IN RESPIRATORY PATIENTS}

${ }^{1} \mathrm{~S}$ Gonem, ${ }^{2} \mathrm{~A}$ Taylor, ${ }^{2} \mathrm{G}$ Figueredo, ${ }^{2} \mathrm{~S}$ Forster, ${ }^{2} \mathrm{~T}$ McKeever, ${ }^{2} \mathrm{~S}$ Garibaldi, ${ }^{2} \mathrm{D}$ Shaw. ${ }^{1}$ Nottingham University Hospitals NHS Trust, Nottingham, UK; ${ }^{2}$ University of Nottingham, Nottingham, UK

\subsection{6/thorax-2021-BTSabstracts.23}

Background The National Early Warning Score-2 (NEWS2) is used to detect patient deterioration in UK hospitals but relies on a snapshot assessment. We developed a Dynamic Early Warning Score (DEWS) which takes into account changes over time, and assessed its accuracy in respiratory patients for predicting death or intensive care unit (ICU) admission, both within 24 hours, and clinical deterioration within 4 hours.
Methods Clinical observations data were extracted from electronic data records for 31590 respiratory in-patient episodes (53.5\% female, mean age 69.9) at a large acute NHS Trust from $1^{\text {st }}$ April 2015 to $31^{\text {st }}$ December 2020, comprising 1037349 date and time-stamped observation sets. 786 inpatient episodes comprising 52300 observation sets were annotated manually following reference to the medical case-notes. Clinical deterioration was defined as a specific event (eg. hospital-acquired pneumonia) requiring a change in treatment (eg. antibiotics). Development of DEWS used similar methodology to Zhu et al (Resuscitation. 2020; 157: 176-84). Continuous variables were divided into two separate variables corresponding to high or low values. Time series features including rolling average, standard deviation and trend over previous observations were entered into a logistic regression model. For death or ICU admission within 24 hours, data from 2015-2019 and 2020 were used for model training and validation respectively. For clinical deterioration within 4 hours, Results presented are for the training dataset (validation dataset is pending).

Results Death or ICU admission occurred within 24 hours of $2.3 \%$ of observations sets. The area under the receiver operating curve (AUC $[95 \%$ confidence interval]) for predicting death or ICU admission within 24 hours was 0.903 (0.897 $0.911)$ for DEWS versus $0.862(0.859-0.865)$ for NEWS2 in the training dataset, and $0.901(0.892-0.908)$ for DEWS versus $0.854(0.849-0.858)$ for NEWS2 in the validation dataset. Clinical deterioration occurred within 4 hours of $6.6 \%$ of observation sets. The AUC for predicting clinical deterioration within 4 hours was $0.861(0.842-0.878)$ for DEWS versus $0.793(0.783$ - 0.801) for NEWS2.

Conclusions DEWS has superior performance compared to NEWS2 with respect to predicting death or ICU admission within 24 hours, and clinical deterioration within 4 hours, in respiratory patients.

Please refer to page A188 for declarations of interest related to this abstract.

\section{S18 INVESTIGATING THE IMPACT OF INFLUENZA ACTIVITY ON EXCESS MORTALITY RATES FROM CARDIOVASCULAR, RESPIRATORY AND RENAL DISEASES IN IRELAND DURING THE 2010/11-2019/20 INFLUENZA SEASONS}

${ }^{1,2} \mathrm{JX}$ Choo, ${ }^{2,3} \mathrm{~J}$ Harbison. 'School of Medicine, Trinity College Dublin, Dublin, Ireland; ${ }^{2}$ Mercer's Institute for Successful Ageing, St James's Hospital, Dublin, Ireland; ${ }^{3}$ Department of Medical Gerontology, School of Medicine, Trinity College Dublin, Dublin, Ireland

10.1136/thorax-2021-BTSabstracts.24

Introduction COVID-19 and influenza infection are associated with cardiovascular, respiratory and renal complications. However, cardiovascular, respiratory and renal disease mortality rates in Ireland decreased by $0.04 \%, 0.12 \%$ and $0.12 \%$ respectively in 2020 relative to 2019 , despite nearly 100,000 new COVID-19 cases being reported in Ireland in 2020. Government-imposed social distancing measures resulted in abolition of influenza activity (IA). We analysed population data from the 2010/11-2019/20 influenza seasons to estimate the impact of reduced IA on cardiovascular, respiratory and renal disease mortality rates during the COVID-19 pandemic season. Methods Quarterly mortality data for acute myocardial infarction (AMI), cerebrovascular disease, pneumonia, asthma and renal disease from first quarter (Q1) 2010 to fourth quarter (Q4) 2020 was obtained from the Central Statistics Office. 